\section{Derechos económicos de las mujeres rurales en el proceso de cambio de Bolivia: un análisis normativo}

Bishelly Elías Argandoña

Economista rural e investigadora.

Miembro de la Red de Mujeres

Transformando la Economía, Bolivia.
Economía Social y Solidaria /

Perspectivas

RECEPCIÓN: 18/06/15

ACEPTACIÓN FINAL: 27/07/15

\section{Resumen}

El "proceso de cambio" boliviano que se inicia en el año 2006 configura un nuevo escenario para generar normas que promuevan los derechos económicos de las mujeres rurales. La Constitución Política del Estado plantea la equidad de género como un principio del sistema de gobierno, sin embargo las agendas de las organizaciones de mujeres urbanas y rurales no son las mismas; mientras que las mujeres urbanas han avanzado hacia la consolidación de la participación política, las mujeres rurales han avanzado en los derechos de acceso a la tierra y desarrollo rural. Ambos caminos presentan desafíos para lograr que la participación igualitaria de las mujeres en los distintos niveles de Estado sea una oportunidad para efectivizar los derechos de las mujeres rurales.

\section{Resumo}

O "processo de mudança" iniciado na Bolívia em 2006 estabeleceu um novo estágio para a geração de padrões que promovam direitos econômicos das mulheres rurais. A Constituição Política do Estado gera a equidade de gênero como um princípio de governação, porém as agendas das organizações de mulheres rurais e urbanas não são as mesmas, enquanto as mulheres urbanas têm progredido até a consolidação da participação política, as mulheres rurais têm avançado nos direitos de acesso à terra e desenvolvimento rural. Os dois caminhos apresentam desafios para conseguir que a participação de igualdade das mulheres nos diferentes níveis de Estado seja uma oportunidade para que os direitos das mulheres rurais se tornem mais eficazes.

Palavras-chave

- Processo de mudança

- Bolívia

- mulheres rurais

- direitos das mulheres
Para citación de este artículo

Elías Argandoña, B. (2015). Derechos económicos de las mujeres rurales en el proceso de cambio de Bolivia: un análisis normativo. En Revista +E versión digital, (5), pp. 68-75. Santa Fe, Argentina: Ediciones UNL. 


\section{Presentación}

Bolivia, desde el año 2006, ha dado un giro en la política pública. Durante el período anterior (1985-2005) el motor principal de esta política fue la estabilidad económica y la atracción de inversión extranjera para las empresas públicas existentes. El rol del Estado era otorgar mejores servicios de salud, educación, teniendo muy poco ámbito de acción en el área rural, solamente la ley de Reforma Agraria (INRA) y el fortalecimiento de la descentralización administrativa a nivel municipal, como políticas de ámbito nacional, con algunos avances en derechos específicos de las mujeres. ${ }^{1}$ A partir del denominado "proceso de cambio" (2006 en adelante) el Estado asume un rol protagónico con la Nueva Constitución Política (2008), en la que se establece la inclusión de los saberes de los pueblos indígenas bolivianos que conforman la mayoría de la población y se promueve, al menos normativamente, el proceso autonómico departamental, municipal e indígena. En el nivel económico, el Estado asume un rol central a través de la nacionalización de recursos y empresas estratégicas (hidrocarburos, telecomunicaciones, entre otras) y la creación de nuevas empresas públicas, así como la promoción de la inversión pública a nivel urbano y rural para fomentar el crecimiento. En este proceso, hay ciertos temas que debido a su complejidad es difícil de analizar y que, pese a que estamos ya a nueve años bajo esta nueva dirección, todavía no se vislumbran resultados prácticos en el apoyo a las mujeres rurales. Existen avances en cuanto a la visión y participación política del Estado y de las mujeres en la política a través de la paridad y alternancia. Existen cambios significativos en las políticas de desarrollo rural, donde las más importantes son la efectivización de la titulación de tierras para hombres y mujeres, la ley de la revolución productiva y de la agricultura familiar. Existen, a su vez, avances en cuanto a la generación de un plan de igualdad de oportunidades a nivel nacional y existen la ley contra el racismo y toda forma de discriminación, así como la ley contra la violencia hacia la mujer, que al menos normativamente se intenta que llegue a cumplirse en los distintos ámbitos.

Aun así, es difícil ver a las mujeres como actoras económicas, como agricultoras, comerciantes, trabajadoras agrarias y no agrarias, como las trabajadoras de su hogar, como las responsables de la crianza de la vida dentro y fuera de sus parcelas. En este sentido es que, aunque existen avances normativos, todavía falta la exigibilidad desde los grupos de mujeres rurales y la voluntad política para que se pongan en práctica.
El artículo comprenderá los principales cambios normativos que ha vivido Bolivia desde la Constitución Política del Estado: las leyes de apoyo al desarrollo rural, la ley de acceso a la tierra (Ley INRA) y, por otro lado, las leyes que van en busca de la igualdad de género, ambas siguen su propio camino, a veces paralelo, pocas veces intersectado, pero que no se vislumbra como un aporte efectivo a generar cambios en la vida de las mujeres rurales.

\section{Los grandes cambios: la Constitución Política del Estado} La Constitución Política del Estado Plurinacional de Bolivia ha incorporado varios artículos que priorizan la igualdad de oportunidades entre hombres y mujeres, tanto en la participación política, económica, como social y el acceso a los servicios públicos y privados con equidad y sin discriminación. El artículo 8 señala como un valor la igualdad de oportunidades y la equidad social y de género en la participación. El artículo 11 indica que Bolivia adopta para su gobierno la forma democrática participativa, representativa y comunitaria, con equivalencia de condiciones entre hombres y mujeres. El artículo 270 define los principios que rigen la organización territorial del Estado y las entidades territoriales descentralizadas y autónomas, entre ellos: bien común, solidaridad, autogobierno, igualdad, equidad de género, transparencia, participación y control social. De esta manera, a nivel de valores y principios, la equidad de género está garantizada en la CPE.

En los artículos 13 y 14 se prohíbe y sanciona toda forma de discriminación en razón de sexo, edad, cultura o cualquier otra condición, por afectar el reconocimiento, goce o ejercicio de los derechos de toda persona, en condiciones de igualdad; el artículo 15 contempla el derecho de las mujeres a vivir libres de violencia en la familia y en la sociedad y el compromiso del Estado de adoptar todas las medidas necesarias para eliminar y sancionar la violencia de género. En el artículo 26 se define que la participación política será equitativa y en igualdad de condiciones entre hombres y mujeres. En el artículo 147 se señala que en la elección de asambleístas se garantizará la igual participación de hombres y mujeres y en el 278 se expresa que la ley determinará los criterios generales para la elección de asambleístas departamentales, tomando en cuenta la paridad y alternancia de género. De este modo se establece y define la paridad y alternancia en la participación política y se señala la realización de una ley específica que garantice este derecho.
1) Los principales avances en derechos de las mujeres en el período 1985-2005 se dan en la concreción de derechos en salud, educación, familiares y temas laborales. De manera específica se tiene la aprobación del convenio sobre la Eliminación de Todas las Formas de Discriminación Contra la Mujer, las cuotas de participación política y el derecho de acceso a la tierra. 


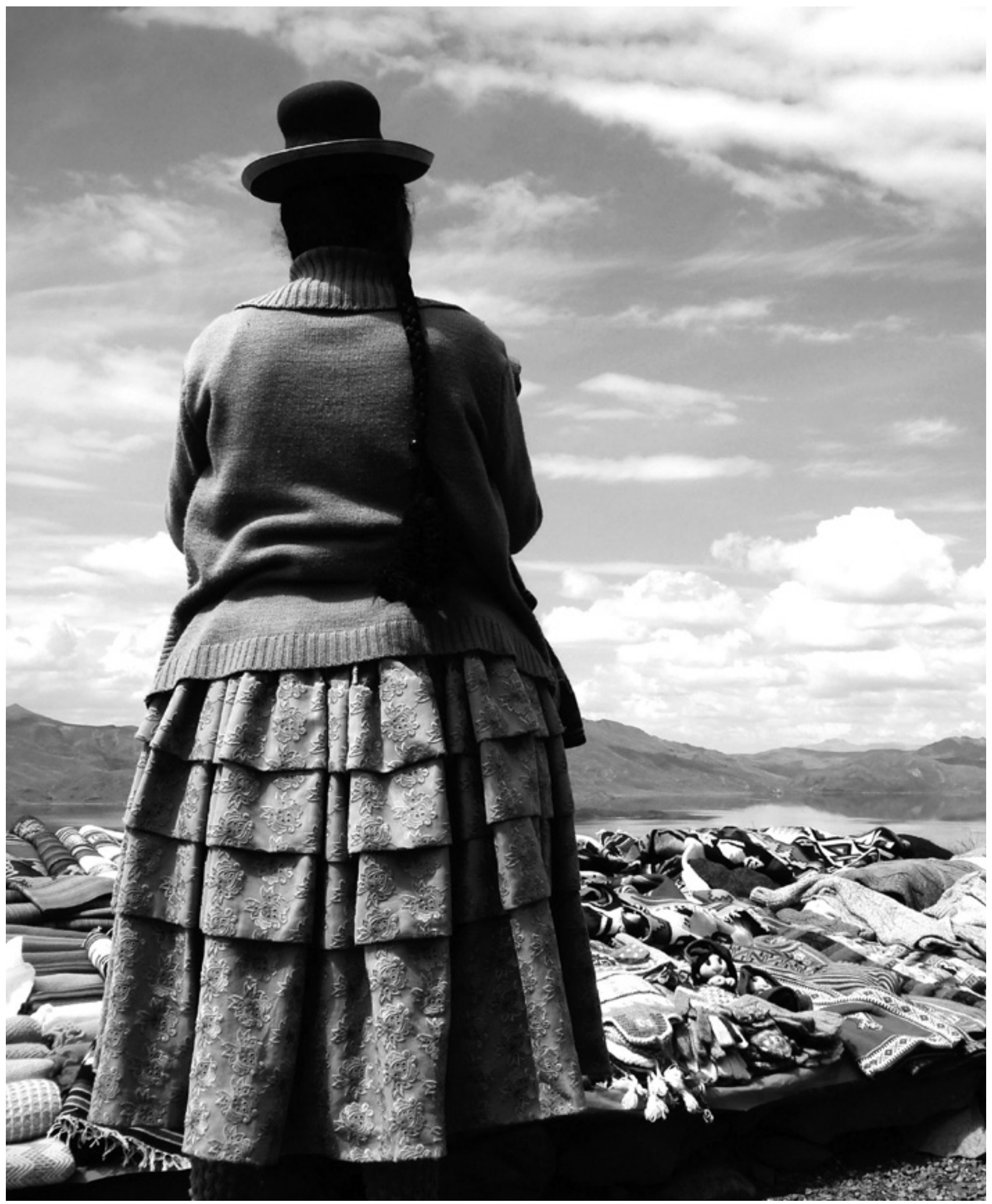


El artículo 82 garantiza la eliminación de las discriminaciones de género existentes en el ámbito de educación, que dificultan la entrada y afectan el desempeño de muchas mujeres en los sectores de la economía y el 62 reconoce que todos los integrantes de la familia tienen igualdad de derechos, obligaciones y oportunidades. Y señala, por un lado, la posibilidad de realizar acciones afirmativas en cuanto a educación para las mujeres y, por otro, la corresponsabilidad existente en el ámbito familiar para el cumplimiento de las distintas actividades en el ámbito privado. El desarrollo rural integral sustentable se establece en el Título Tercero con cinco artículos (405-409) en los que se define que es parte fundamental de las políticas económicas del Estado, que priorizará sus acciones para el fomento de todos los emprendimientos económicos comunitarios y del conjunto de los actores rurales, con énfasis en la seguridad y en la soberanía alimentaria (art. 405). El artículo 406 define que el Estado garantizará el desarrollo rural integral sustentable por medio de políticas, planes, programas y proyectos integrales de fomento a la producción agropecuaria, artesanal, forestal y al turismo, que se promoverá y fortalecerá a las organizaciones económicas productivas rurales, entre ellas a los artesanos, las cooperativas, las asociaciones de productores agropecuarios y manufactureros, y las micro, pequeñas y medianas empresas comunitarias agropecuarias.

En el artículo 407 se establece que se garantizará la soberanía y seguridad alimentaria, priorizando la producción y el consumo de alimentos de origen agropecuario producidos en el territorio boliviano. En el 408 el Estado determina estímulos en beneficio de los pequeños y medianos productores con el objetivo de compensar las desventajas del intercambio inequitativo entre los productos agrícolas y pecuarios con el resto de la economía, y en el 409 se señala que la producción, importación y comercialización de transgénicos será regulada por ley. Sin embargo, en ninguno de los artículos se habla del rol fundamental que tienen las mujeres rurales en el desarrollo rural integral sustentable ni se dice que se promoverá y/o facilitará su participación.

\section{La ley por la tierra, la Ley INRA}

La Ley INRA es del año 1996 y establece el Servicio Nacional de Reforma Agraria y el régimen de distribución de tierras; procura garantizar el derecho propietario sobre la tierra; crea la Superintendencia Agraria, la Judicatura Agraria y su procedimiento, y regula el saneamiento de la propiedad agraria.

En el artículo 3, de Garantías constitucionales, se señala que el Servicio Nacional de Reforma Agraria, en concordancia con el artículo $6^{\circ}$ de la Constitución Política del Estado y en cumplimiento de las disposiciones contenidas en la Convención sobre la Eliminación de todas las Formas de Discriminación contra la Mujer, aplicará criterios de equidad en la distribución, administración, tenencia y aprovechamiento de la tierra en favor de la mujer, independientemente de su estado civil. No obstante, pese a este artículo, la práctica de la dotación entre 1996 y 2005 nos muestra que la mayor parte de títulos a mujeres es para mujeres solas, aunque con gran desigualdad respecto de los varones, ya que por cada título para mujer que se entregaban cuatro a varones solos $y$, en el caso de que la tierra fuera de la pareja, el título aparecía a nombre sólo del varón.

La Ley N³545, de Reconducción Comunitaria de la Reforma Agraria, de noviembre de 2006, fue una de las primeras leyes para el sector rural en el proceso de cambio y señala en la disposición final octava (equidad de género) que se garantiza y prioriza la participación de la mujer en los procesos de saneamiento y distribución de tierras. En caso de matrimonios y uniones conyugales libres o de hecho, los títulos ejecutoriales serán emitidos a favor de ambos cónyuges o convivientes que se encuentren trabajando la tierra, consignando el nombre de la mujer en primer lugar. Igual tratamiento se otorgará en los demás casos de copropietarios mujeres y hombres que se encuentren trabajando la tierra, independientemente de su estado civil. Este derecho especificado en la norma, sumado a la voluntad política del Instituto Nacional de Reforma Agraria de lograr que las mujeres lo ejerzan, ha cambiado en el período 2005-2014 la titulación de la tierra a nombre de las mujeres, de forma que ha disminuido la 
brecha entre hombres solos y mujeres solas, de 4 a 3 títulos para varones por cada título para mujer (Elías, 2013), pero sobre todo ha logrado la incorporación de las mujeres en los títulos de pareja que, de acuerdo con el Informe Presidencial de 2013, son el $47 \%$ de las beneficiarias.

Aun así, quedan algunas brechas por trabajar; por ejemplo, se ha identificado que el tamaño de la tierra de las mujeres es menor que el de la que corresponde a los varones, o que en las propiedades colectivas, como los Territorios Comunitarios de Origen (TCO), no existen normas claras para la dotación de tierra a las mujeres.

\section{La normativa para el desarrollo rural}

La Ley de Revolución Productiva, de junio de 2011, tiene por objeto normar el proceso de Revolución Productiva Comunitaria Agropecuaria, para la soberanía alimentaria y establece las bases institucionales, políticas y mecanismos de la producción, transformación y comercialización de productos agropecuarios y forestales, de los diferentes actores de la economía plural; priorizando la producción orgánica en armonía y equilibrio con las bondades de la madre tierra. La norma, sin embargo, no indica en ninguno de los artículos cuál es la participación de la mujer en este proceso y solamente en los principios (artículo 6) se señala que la alimentación adecuada es un principio que no discrimina clase social, credo religioso, opción política, género y generacional. De esta manera la norma más importante para el desarrollo rural, y que pudo haber abierto reconocimiento de la mujer rural como una actora económica importante del mismo, simplemente no la visibiliza como tal.
La Ley $N^{\circ} 338$, de Organizaciones Económicas Campesinas, Organizaciones Económicas Comunitarias y de Agricultura Familiar, de enero de 2013, tiene por objeto normar

"la agricultura familiar sustentable y las actividades familiares diversificadas, realizadas por las Organizaciones Económicas Campesinas, Indígena Originarias (OECAS), las Organizaciones Económicas Comunitarias (OECOM), y las familias productoras (...) basadas en el uso y aprovechamiento de los componentes de la Madre Tierra (...) para contribuir a la soberanía alimentaria." (art. 2).

Esta norma asume como principio (art. 7) la obligación de impulsar acciones que garanticen equidad de género, que donde prima la familia compuesta por hombres, mujeres, jóvenes y adultos mayores, éstos se reconocen en sus diferencias y complementariedades, participan en igualdad y contribuyen con equidad para el desarrollo social, económico y productivo para el Vivir Bien, y reconoce el aporte productivo de la mujer rural para la producción de alimentos destinados al consumo nacional y la soberanía alimentaria, a la generación de excedentes y al desarrollo del sector productivo. De forma que enmienda los olvidos de la anterior norma.

En el artículo 17, de Obligaciones, indica como obligación del Estado Plurinacional de Bolivia:

- visibilizar el rol de las mujeres y de la juventud rural en la agricultura familiar sustentable;

- el Instituto Nacional de Estadística (INE) deberá generar información oficial del aporte desagregado de hombres y mujeres en la agricultura familiar sustentable;

- el nivel central del Estado y las entidades territoriales autónomas 


\section{6}

\section{se ha efectivizado la participación política de la mujer y, a 2015, tenemos en la Asamblea Legislativa una participación del $50 \%$ de mujeres, y en los municipios un $51 \%$ de concejalas mujeres}

promoverán el efectivo acceso de las mujeres a los componentes de producción en igualdad de oportunidades y condiciones, en especial, a créditos productivos de fomento y a la educación técnica y tecnológica especializada.

En el artículo 38 se señala la existencia de igualdad de oportunidades y democratización en el acceso a financiamiento para emprendimientos productivos; en el artículo 14 se promueve la participación de mujeres y hombres en igualdad de oportunidades y funciones en las directiva de las OECA y en el 34, que define los actores que participan en el control social, establece que se debe garantizar la participación de las organizaciones de mujeres.

De estas distintas directrices se ha consolidado en otra normativa la de facilitar el acceso al crédito de las mujeres a través del Decreto Supremo $N^{\circ} 2310$ de marzo de 2015, en el que se expresa que las mujeres que desarrollan actividades en el sector productivo, en áreas rural y urbana, podrán acceder a préstamos bajo el producto denominado "Crédito Productivo Grupal para la Mujer" a través de un fideicomiso ya establecido por ley.

\section{La normativa de igualdad}

El Decreto Supremo № 29850, de diciembre de 2008, aprueba el Plan Nacional para la Igualdad de Oportunidades (PNIO) "Mujeres Construyendo la Nueva Bolivia para Vivir Bien", que se constituye como un mandato para la incorporación del enfoque de género en las instituciones públicas. Adicionalmente a los derechos universales, como salud, educación, se establecen los ejes de participación política, violencia de género y derechos económicos productivos y laborales de los mujeres a fin de que alcancen mayor autonomía económica.

En el eje económico productivo y laboral se establecen como políticas: - promover el ejercicio de los derechos laborales de las mujeres y el acceso al trabajo digno;

- promover el acceso de las mujeres a los recursos naturales, a los recursos productivos y a los servicios públicos;

- promover el acceso de las mujeres al patrimonio tangible (tierra, vivienda, capital) e intangible (tecnología, capacitación); y

- redistribuir las tareas del cuidado y protección de la familia entre mujeres y hombres dentro del hogar y entre las familias y el Estado.

Estas políticas deben ser implementadas por distintos Ministerios $y$, sin embargo, seis años después, no se conoce cómo estas instituciones han puesto en práctica estas políticas.

A nivel de participación política, la ley del Régimen Electoral, de junio de 2010, en su artículo 11 (equivalencia de condiciones), dice que

"la democracia intercultural boliviana garantiza la equidad de género y la igualdad de oportunidades entre mujeres y hombres. Las autoridades electorales respetarán la paridad y alternancia de género entre mujeres y hombres, de tal manera que (...) exista una candidata titular mujer $y$, a continuación, un candidato titular hombre; un candidato suplente hombre y, a continuación, una candidata suplente mujer, de manera sucesiva."

Así se ha efectivizado la participación política de la mujer y, a 2015, tenemos en la Asamblea Legislativa una participación del $50 \%$ de mujeres, y en los municipios un $51 \%$ de concejalas mujeres. 
Otras normas importantes que se han desarrollado en estos años son la ley contra el acoso y la violencia política hacia las mujeres (Ley $\mathrm{N}^{\circ} 243$ ), la ley contra el racismo y toda forma de discriminación (Ley $N^{\circ}$ 045) y la ley integral para garantizar a las mujeres una vida libre de violencia (Ley $\mathrm{N}^{\circ} 348$ ), todas ellas tienen por objeto establecer mecanismos, medidas y políticas integrales de prevención, atención, protección y reparación a las mujeres en situación de violencia, acoso y/o discriminación.

En los últimos años, en el marco de la nueva Constitución se ha desarrollado una serie de normas que buscan garantizar derechos fundamentales para las mujeres y una participación política equitativa. Existen todavía bastantes propuestas desde distintas organizaciones de mujeres para garantizar derechos fundamentales para las mujeres, como la despenalización del aborto y otras de carácter estructural, como la despatriarcalización, que esperemos se vayan a incorporar en la normativa nacional.

\section{Las mujeres detrás de estos procesos}

Cada una de las normas citadas ha tenido un proceso histórico propio. En la normativa para la igualdad de oportunidades, participación política, existe una deuda de la sociedad boliviana hacia los distintos movimientos, agrupaciones y organizaciones de mujeres, principalmente urbanas, pero cada vez con mayor participación de mujeres del área rural que han logrado consolidar sus propuestas. En los casos de violencia, acoso y/o discriminación, les debemos la movilización a estos mismos movimientos y fundamentalmente a los casos públicos de las mujeres víctimas que han muerto en manos de sus agresores. ${ }^{2}$ La normativa de desarrollo rural y tierras ha sido dialogada y desarrollada en conjunto con distintas organizaciones campesinas e indígenas: con la Confederación Sindical Única de Trabajadores Campesinos de Bolivia (CSUTCB), la Confederación Sindical de Interculturales de Bolivia (CSIB) que son organizaciones articuladas a nivel nacional y de las distintas regiones y la Confederación Nacional de Mujeres Campesinas Indígenas Originarias de Bolivia, Bartolina Sisa (CNMCIOB BS) ${ }^{3}$ especialmente, aunque han participado muchas más organizaciones en este proceso. "Las Bartolinas" ha sido de las pocas organizaciones de mujeres rurales, si no la única, que ha participado en la definición de políticas de desarrollo rural. También algunas representantes de esta organización han pasado a ocupar cargos públicos importantes. Sin embargo, su participación y propuesta no se han enfocado en las necesidades y demandas de las mujeres rurales sino en las del área rural en general, y aunque ellas han sido actoras principales en la generación de una propuesta de ley marco para la soberanía alimentaria, por ejemplo, ésta no llego a ser priorizada por el gobierno nacional y fue dejada de lado. En algunos estudios (Elías, 2013 y Dorrego y Elías, 2014), cuando se pregunta a las mujeres rurales si conocen alguna política de apoyo hacia las mujeres, les es muy difícil identificarlas; algunas han reconocido que se han hecho "promesas" pero no políticas con las que se sientan favorecidas. Después de la promulgación de la ley integral para garantizar a las mujeres una vida libre de violencia ésta es la norma más reconocida como de "apoyo hacia la mujer".

\section{Conclusiones}

La configuración del nuevo Estado boliviano ha dado la oportunidad de que se puedan readecuar o rehacer distintas normativas de forma que sean incluyentes y efectivas. La CPE deja abierto el marco para promover la igualdad de oportunidades y la equidad de género en distintos ámbitos, y quedan a merced de las voluntades políticas y las exigencias de la sociedad civil los principales caminos a seguir. En esto años se observa que las prioridades para los diversos sectores son distintas. Las organizaciones de mujeres han priorizado la agenda de participación política para garantizar derechos fundamentales de las mujeres, mientras que las organizaciones rurales, incluidas las mujeres rurales, han priorizado una agenda para garantizar el acceso a recursos (tierra) y la promoción del desarrollo productivo de manera general.

Los derechos de las mujeres rurales muestran avances en cuanto al acceso a los recursos, en este caso "tierra", sin embargo continúan las brechas existentes entre hombres y mujeres y no se muestran avances significativos en cuanto al acceso a otro tipo de derechos, tales como el acceso a recursos productivos.

Existen algunos encuentros esporádicos entre estos dos movimientos, en algunos casos las mujeres rurales que incluyen entre sus
2) El caso más emblemático es el de la periodista Hanali, que fue asesinada por su esposo el 13 de febrero de 2013, y la ley fue aprobada el 9 de marzo del mismo año.
3) Estas tres organizaciones son reconocidas como las que han fundado el instrumento político para la soberanía de los pueblos IPSP-MAS, partido que lleva cabo "el proceso de cambio" en funciones de gobierno desde 2006. 


\section{G6}

las organizaciones de mujeres han priorizado la agenda de participación política para garantizar derechos fundamentales de las mujeres, mientras que las organizaciones rurales, incluidas las mujeres rurales, han priorizado una agenda para garantizar el acceso a recursos (tierra) y la promoción del desarrollo productivo de manera general

demandas una vida libre de violencia y conocen la normativa para poder exigirla, en otros casos organizaciones urbanas que trabajan con mujeres rurales por el reconocimiento y la exigibilidad de su derecho a la tierra. Pero queda un amplio espectro de acción para el reconocimiento de los derechos de las mujeres rurales, y aquí pueden aprender mucho del camino recorrido por las organizaciones de mujeres urbanas. Algunas normas representan ya grandes avances, específicamente la Ley de Organizaciones Económicas Campesinas, Organizaciones Económicas Comunitarias y de Agricultura Familiar o el Plan Nacional de Igualdad de Oportunidades, aunque deben exigir su cumplimiento.

Es importante que las organizaciones de mujeres rurales planteen su propia agenda en este "proceso de cambio" que ciertamente puede tener muchos aspectos en común con la de sus pares varones pero con sus propias especificidades. Algunas de estas demandas son el derecho a la soberanía alimentaria, el acceso a otros recursos importantes, como el agua y semillas propias, apoyos específicos para los sistemas de producción y comercialización, que tienen el reconocimiento del patrimonio ambiental y cultural que ellas preservan, incluyendo sus propios saberes, la corresponsabilidad, entre otros.
¿Podrán las mujeres rurales incluir su agenda en el "proceso de cambio"? La participación igualitaria en los distintos niveles de Estado es una oportunidad para que sus propuestas puedan efectivizarse. Esto implica un trabajo de incidencia en decisoras políticas y plantea la posibilidad de tender lazos entre mujeres de distintas organizaciones, rurales y urbanas, para que sus derechos económicos no sean postergados.

\section{Referencias bibliográficas}

Dorrego, A. y Elías, B. (2014). Agroecología y comercialización campesina. En Cuaderno de Trabajo. Bolivia: REMTE (19), 55.

Elías, B. (2013). La soberanía alimentaria desde las mujeres. En Cuaderno de Trabajo. Bolivia: REMTE (19), 55. 